\title{
Characteristics of Australian Osteopaths Managing Tendinopathies: Analysis of a Nationally Representative Survey of 989 Practitioners
}

\author{
B. Vaughan ${ }^{1,2}$, M. Fleischmann ${ }^{3}$, J. Mest ${ }^{4}$, K. Fitzgerald ${ }^{5}$, P. Malliaris ${ }^{6}$ \\ 1 Department of Medical Education, University of Melbourne, Melbourne, Australia \\ 2 School of Health \& Human Sciences, Southern Cross University, Lismore, Australia \\ 3 College of Health \& Biomedicine, Victoria University, Melbourne, Australia \\ ${ }^{4}$ University of Canberra Health Clinics, Faculty of Health, University of Canberra, Canberra, Australia \\ 5 School of Health \& Biomedical Sciences, RMIT University, Melbourne, Australia \\ 6 Department of Physiotherapy, School of Primary and Allied Health Care, Monash University, Melbourne, \\ Australia
}

\section{CORRESPONDING AUTHOR:}

\section{Brett Vaughan}

Department of Medical Education

Melbourne Medical School

University of Melbourne

Grattan Street, Melbourne, Australia

E-mail: brett.vaughan@unimelb.edu.au

DOI:

10.32098/mltj.01.2021.16

LEVEL OF EVIDENCE: 4

\begin{abstract}
SUMMARY
Background. Tendinopathies are a common complaint presenting to health professionals, including osteopaths. Evidence supports the use of exercise rehabilitation, with a potential role for manual therapy. This study sought to profile the practice and clinical management characteristics of Australian osteopaths who often treat tendinopathies. Methods. The study was designed as a cross-sectional survey of Australian osteopaths. Participants were Australian osteopaths who were members of the Osteopathy Research and Innovation Network (ORION) - the Australian professions' practice-based research network. Participants were invited to complete a 27 -item practice and clinical management characteristic questionnaire. The questionnaire asked participants to identify demographic, practice and clinical management characteristics associated with their practice. Regression modelling was used to identify significant characteristics associated with often treating tendinopathies.

Results. Nine hundred and eighty-nine osteopaths responded and over $41 \%$ ( $\mathrm{n}=$ 410) reported often treating tendinopathies. This group were more likely to be female (ORa 1.5), often treat patients with chronic pain (ORa 3.8) and sports injury (ORa 2.5) and often use exercise prescription (ORa 1.6) in patient care.

Conclusions. Australian osteopaths who often treat tendinopathies demonstrate different clinical practice and management characteristics compared to their colleagues who do not often treat this complaint. Research into patient outcomes and cost-effectiveness of osteopathy care for the management of tendinopathies is now required.
\end{abstract}

\section{KEY WORDS}

Musculoskeletal; manual therapy; rebabilitation; osteopathic medicine; practice-based research network.

\section{INTRODUCTION}

Tendons are a unique connective tissue attaching muscle to bone (1) and loading the tendon beyond its load capacity has been identified as a key risk factor to the development of tendinopathy (2). Tendinopathy is a highly prevalent condition that affects people from both athletic and non-athletic backgrounds (3-6). Prevalence studies across a variety of contexts suggests that tendinopathies are likely to be a common presentation to a range of health professionals (7). Much of the prevalence literature focuses on lower extremity tendinopathies in different age groups (8), and populations (3), including those competing in sport at the elite (3) and non-elite level (9). However, Hopkins et al. (10) argue the prevalence of tendinopathies may be underestimated. First line management of tendinopathy is exercise-based (2). Contemporary narrative suggests that progressively expos- 
ing the tendon to heavy slow resistance (HSR), the best way to: 1) improve load tolerance and mechanical stiffness of the tendon being loaded (2) and 2) induce adaptive changes to the musculotendinous unit. Loading programs have been shown to be efficacious in the management of adults with lower limb tendinopathy $(11,12)$.

There is an emerging body of evidence that draws associations between tendinopathy and psychosocial factors (13). Kinesiophobia or fear of movement was found to be present in 2 of every 3 patients in a consecutive cohort of adults with lower limb tendinopathy. Kinesiophobia, catastrophisation and depression were found to be positively associated with plantar heel pain (14) and with increased symptom severity in gluteal tendinopathy (15). When treating tendinopathy, evidence-based approaches to addressing psychosocial issues and chronic pain management may also be required as part of a multifaceted, patient-centred approach.

Osteopathy offers a whole person approach utilising a patient-centred, biopsychosocial (BPS) model. As such, osteopaths are well placed to play a role in the multidisciplinary management of musculoskeletal conditions including tendinopathy. Australian osteopaths are primary contact health professionals who utilise a range of manual therapy and exercise interventions in patient care, in addition to advice with respect to nutrition, stress and ergonomics (16). In Australia, osteopaths are not trained medical professionals but are able to examine and diagnose conditions affecting the musculoskeletal system, including tendinopathies. Recent workforce data suggests osteopaths manage tendinopathies relatively frequently (16) and are well placed to institute best practice tendinopathy management. The aim of this study was to profile the practice and clinical management characteristics of Australian osteopaths who often manage tendinopathies to better understand the role these health professions play in its management.

\section{METHODS}

Data for the secondary analysis were drawn from the practice-based research network (PBRN) established for the osteopathy profession in Australia - the Osteopathy Research and Innovation Network (ORION) Project (http://www. orion-arccim.com/) (16).

\section{Sample}

Nine hundred and ninety-two osteopaths ( $\mathrm{N}=992)$ completed the ORION survey representing $49.1 \%$ of the profession at time of completion. The ORION research design and baseline data have been described elsewhere (16). The
ORION practitioner sample is nationally representative of the wider profession on a number of indicators including age, gender and primary practice location by comparison with data from the Australian Health Practitioner Regulation Agency (16).

\section{Questionnaire}

Participants completed a questionnaire designed to collect demographic characteristics, practice characteristics, and clinical management of patient's via 27 items. Demographic characteristics included age, gender, the highest level of osteopathy professional qualification, and length of time working in private osteopathy practice. Practice characteristics include the average patient care hours and patient visits per week, practice location, health professionals working in same practice location, referral relationships (receiving and sending) with other health professionals, and diagnostic imaging use. The participants were asked about their clinical management, such as people' conditions, patient groups, and various aspects of osteopathy techniques. With respect to the current study, respondents were asked to indicate the frequency with which they manage patients with a tendinopathy (never/rarely/sometimes/often) as the outcome variable.

\section{Statistical analyses}

Participants were categorised as based on their response to the frequency item. For the analysis, the outcome variable was dichotomised to 'not often' (never/rarely/sometimes) and 'often' treating patients with tendinopathies. Participants' demographics, practice characteristics, and clinical management were then compared between groups via chi-square tests and independent measures t-tests. Unadjusted odds ratios (OR) were calculated for the chi-square tests, and effect sizes calculated for t-tests where relevant. Variables with a $\mathrm{p}<0.10$ were subsequently included in a multiple logistic regression model, using a backward stepwise elimination process, to examine the practice characteristics statistically significantly associated with frequency of managing tendinopathies. Alpha was set at 0.05 and adjusted odds ratios $(\mathrm{ORa})$ were estimated from this multivariate logistic regression modelling. All statistical analyses were performed using SPSS version 25.

\section{Ethical approval}

Osteopaths who chose to participate in the PBRN provided informed consent and ethics approval was granted through the University of Technology Sydney (approval number: 
2014000759). The data collection was performed in accordance with the ethical standards for the journal described by Padulo et al. (17).

\section{RESULTS}

Nine hundred and eighty nine respondents $(99.7 \%$ of the ORION database) provided a response to the frequency item "How often do you treat patients with a tendinopathy?" (never/rarely/sometimes/often). The number of Australian osteopaths who reported treating tendinopathies often was $410(41.4 \%)$ (table I). Descriptive statistics for the sample are presented in table I. Australian osteopaths who often treated patients with tendinopathies were $60 \%$ more likely to be female in addition to reporting a greater number of patient visits and patient care hours per week, and being less likely to be involved in research (table I).

Australian osteopaths who often reported treating tendinopathies were more likely to send referrals to, and receive referrals from, a range of health professionals when compared to those who do not (table II). Referrals for diagnostic imaging were also three times more likely to be used by those osteopaths often treating tendinopathies compared to those who do not. Australian osteopaths who report often treating tendinopathies were more likely, compared to those who do not, to discuss a range of lifestyle and self-management strategies with patients, along with treating the breadth of body regions (table III). Most of the common manual therapy techniques utilised by Australian osteopaths were more likely to be used by those reporting often treating tendinopathies compared to those who do not.

Table I. Practitioner characteristics of Australian osteopaths who often treat patients with tendinopathies.

\begin{tabular}{|c|c|c|c|c|}
\hline & 'Often' $(n=410)$ & 'Not often' $(n=579)$ & p-value & OR [95\% CI] \\
\hline \multicolumn{5}{|l|}{ Gender } \\
\hline Male & $210(51.2 \%)$ & $366(63.2 \%)$ & & \\
\hline Female & $200(48.8 \%)$ & $213(36.8 \%)$ & $<0.01$ & $1.6[1.2,2.1]$ \\
\hline \multicolumn{5}{|l|}{ Age (years) } \\
\hline Mean $( \pm \mathrm{SD})$ & $38.3( \pm 10.4)$ & $37.8( \pm 11.2)$ & 0.48 & - \\
\hline \multicolumn{5}{|l|}{ Years in clinical practice } \\
\hline Mean $( \pm \mathrm{SD})$ & $11.8( \pm 8.5)$ & $11.1( \pm 9.3)$ & 0.24 & - \\
\hline \multicolumn{5}{|l|}{ Patient care hours per week } \\
\hline Mean $( \pm \mathrm{SD})$ & $30.3( \pm 11.8)$ & $26.3( \pm 12.0)$ & $<0.01^{\mathrm{a}}$ & - \\
\hline \multicolumn{5}{|l|}{ Patient visits per week } \\
\hline Mean $( \pm$ SD $)$ & $39.9( \pm 18.5)$ & $33.9( \pm 18.3)$ & $<0.01^{\mathrm{b}}$ & - \\
\hline \multicolumn{5}{|l|}{ Qualification (n, \%) } \\
\hline Diploma & $28(6.8 \%)$ & $33(5.7 \%)$ & 0.40 & - \\
\hline Advanced Diploma & $1(0.2 \%)$ & $8(1.4 \%)$ & & \\
\hline Bachelor degree & $91(22.2 \%)$ & $127(21.9 \%)$ & & \\
\hline Master's degree & $283(69.0 \%)$ & $396(68.4 \%)$ & & \\
\hline $\mathrm{PhD}$ & $1(0.2 \%)$ & $4(0.7 \%)$ & & \\
\hline Other & $6(1.5 \%)$ & $11(1.9 \%)$ & & \\
\hline Additional qualification* & $7(1.7 \%)$ & $8(1.4 \%)$ & 0.68 & - \\
\hline \multicolumn{5}{|l|}{ Involved in as an osteopath } \\
\hline University teaching & $49(12.0 \%)$ & $67(11.6 \%)$ & 0.85 & - \\
\hline Clinical supervision & $66(16.1 \%)$ & $84(14.5 \%)$ & 0.49 & - \\
\hline Professional organisations & $40(9.8 \%)$ & $67(11.6 \%)$ & 0.36 & - \\
\hline Research & $14(3.4 \%)$ & $40(6.9 \%)$ & 0.02 & $0.5[0.2,0.8]$ \\
\hline Volunteer & $66(16.1 \%)$ & $92(15.9 \%)$ & 0.93 & - \\
\hline
\end{tabular}

"Qualification beyond primary osteopathy qualification, ${ }^{\mathrm{a}} d=0.33,{ }^{\mathrm{b}} d=0.32$ (d=Cohen's $d$ effect size). 
Table II. Practice characteristics of Australian osteopaths who often treat patients with tendinopathies.

\begin{tabular}{|c|c|c|c|c|}
\hline & 'Often' (n = 410) & $\begin{array}{c}\text { 'Not } \\
\text { often' }(n=579)\end{array}$ & p-value & OR $[95 \% \mathrm{CI}]$ \\
\hline \multicolumn{5}{|l|}{ Practice location } \\
\hline Urban practice & $336(82.0 \%)$ & $482(83.2 \%)$ & 0.60 & - \\
\hline More than one practice location & $150(36.6 \%)$ & $196(33.9 \%)$ & 0.37 & - \\
\hline Co-located with other health professionals ('yes') & & & & - \\
\hline Osteopath & $275(67.1 \%)$ & $365(63.0 \%)$ & 0.19 & - \\
\hline General Practitioner & $36(8.8 \%)$ & $35(6.0 \%)$ & 0.10 & - \\
\hline Specialist Medical Practitioner & $13(3.2 \%)$ & $18(3.1 \%)$ & 0.95 & - \\
\hline Podiatrist & $71(17.3 \%)$ & $75(13.0 \%)$ & 0.06 & - \\
\hline Physiotherapist & $59(14.4 \%)$ & $85(14.7 \%)$ & 0.90 & - \\
\hline Exercise Physiologist & $57(13.9 \%)$ & $65(11.2 \%)$ & 0.21 & - \\
\hline Occupational Therapist & $5(1.2 \%)$ & $14(2.4 \%)$ & 0.18 & - \\
\hline Psychologist & $78(19.0 \%)$ & $112(19.3 \%)$ & 0.90 & - \\
\hline Massage Therapist & $210(51.2 \%)$ & $289(49.9 \%)$ & 0.68 & - \\
\hline Acupuncturist & $76(18.5 \%)$ & $111(19.2 \%)$ & 0.80 & - \\
\hline Naturopath & $82(20.0 \%)$ & $110(19.0 \%)$ & 0.70 & - \\
\hline Dietician & $29(7.1 \%)$ & $43(7.4 \%)$ & 0.83 & - \\
\hline Nutritionist & $38(9.3 \%)$ & $40(6.9 \%)$ & 0.17 & - \\
\hline \multicolumn{5}{|l|}{ Send referrals to other health professionals ('yes') } \\
\hline Osteopath & $225(54.9 \%)$ & $279(48.2 \%)$ & 0.04 & $1.3[1.0,1.7]$ \\
\hline General Practitioner & $368(89.8 \%)$ & $507(87.6 \%)$ & 0.29 & - \\
\hline Specialist Medical Practitioner & $204(49.8 \%)$ & $237(40.9 \%)$ & $<0.01$ & $1.4[1.1,1.8]$ \\
\hline Podiatrist & $286(69.8 \%)$ & $362(62.5 \%)$ & 0.02 & $1.4[1.0,1.8]$ \\
\hline Physiotherapist & $145(35.4 \%)$ & $186(31.1 \%)$ & 0.29 & - \\
\hline Exercise Physiologist & $186(45.4 \%)$ & $210(36.3 \%)$ & $<0.01$ & $1.5[1.1,1.8]$ \\
\hline Occupational Therapist & $43(10.5 \%)$ & $63(10.9 \%)$ & 0.84 & - \\
\hline Psychologist & $164(40.0 \%)$ & $184(31.8 \%)$ & $<0.01$ & $1.4[1.1,1.8]$ \\
\hline Massage Therapist & $288(70.2 \%)$ & $383(66.1 \%)$ & 0.17 & - \\
\hline Acupuncturist & $182(44.4 \%)$ & $269(46.5 \%)$ & 0.52 & - \\
\hline Naturopath & $203(49.5 \%)$ & $273(47.2 \%)$ & 0.46 & - \\
\hline Dietician & $82(20.0 \%)$ & $84(14.5 \%)$ & 0.02 & $1.5[1.0,2.1]$ \\
\hline Nutritionist & $66(16.1 \%)$ & $63(10.9 \%)$ & 0.02 & $1.6[1.1,2.3]$ \\
\hline \multicolumn{5}{|l|}{ Receive referrals to other health professionals ('yes') } \\
\hline Osteopath & $259(63.2 \%)$ & $353(61.0 \%)$ & 0.48 & $2.0[1.4,2.9]$ \\
\hline General Practitioner & $378(92.2 \%)$ & $505(87.2 \%)$ & 0.01 & $1.7[1.1,2.7]$ \\
\hline Specialist Medical Practitioner & $128(31.2 \%)$ & $108(18.7 \%)$ & $<0.01$ & $2.0[1.5,2.6]$ \\
\hline Podiatrist & $220(53.7 \%)$ & $249(43.0 \%)$ & $<0.01$ & $1.5[1.2,2.0]$ \\
\hline Physiotherapist & $120(29.3 \%)$ & $146(25.2 \%)$ & 0.16 & - \\
\hline Exercise Physiologist & $122(29.8 \%)$ & $135(23.3 \%)$ & 0.02 & $1.4[1.0,1.8]$ \\
\hline Occupational Therapist & $29(7.1 \%)$ & $32(5.5 \%)$ & 0.32 & - \\
\hline Psychologist & $70(17.1 \%)$ & $83(14.3 \%)$ & 0.24 & - \\
\hline Massage Therapist & $326(79.5 \%)$ & $426(73.6 \%)$ & 0.03 & $1.4[1.0,1.9]$ \\
\hline
\end{tabular}




\begin{tabular}{llccc}
\hline & & & \\
& 'Often' (n= 410) & $\begin{array}{c}\text { 'Not } \\
\text { often' (n= 579) }\end{array}$ & p-value & OR [95\% CI] \\
\hline Acupuncturist & $153(37.3 \%)$ & $215(37.1 \%)$ & 0.95 & $1.5[1.1,2.1]$ \\
\hline Naturopath & $183(44.6 \%)$ & $216(37.3 \%)$ & 0.02 & $1.3[1.0,1.7]$ \\
\hline Dietician & $22(5.4 \%)$ & $17(2.9 \%)$ & 0.05 & - \\
\hline Nutritionist & $28(6.8 \%)$ & $27(4.7 \%)$ & 0.14 & - \\
\hline Diagnostic imaging & & & & \\
\hline Referral for imaging ('often') & $49(12.0 \%)$ & $24(4.1 \%)$ & $<0.01$ & $3.1[1.9,5.2]$ \\
\hline Investigation of unknown pathologies & $315(76.8 \%)$ & $427(73.7 \%)$ & 0.27 & - \\
\hline Investigation of suspected diagnosis & $351(85.6 \%)$ & $481(83.1 \%)$ & 0.28 & - \\
\hline Investigation of potential fractures & $318(77.6 \%)$ & $429(74.1 \%)$ & 0.21 & - \\
\hline Rule out risk factors prior to treatment & $130(31.7 \%)$ & $142(24.5 \%)$ & 0.01 & $1.4[1.1,1.9]$ \\
\hline General screening of the spine & $15(3.7 \%)$ & $17(2.9 \%)$ & 0.53 & - \\
\hline Patient assessment ('yes') & & & \\
\hline Orthopaedic testing & $403(98.3 \%)$ & $562(97.1 \%)$ & 0.22 & - \\
\hline Clinical assessment algorithm & $209(51.0 \%)$ & $258(44.6 \%)$ & 0.04 & $1.3[1.0,1.7]$ \\
\hline Neurological testing & $378(92.2 \%)$ & $537(92.7 \%)$ & 0.74 & - \\
\hline Screening questionnaire & $271(66.1 \%)$ & $359(62.0 \%)$ & 0.19 & - \\
\hline Cranial nerve testing & $295(72.0 \%)$ & $375(64.8 \%)$ & 0.02 & $1.4[1.1,1.8]$ \\
\hline
\end{tabular}

Table III. Clinical management characteristics of Australian osteopaths who often treat patients with tendinopathies.

\begin{tabular}{|c|c|c|c|c|}
\hline & $\begin{array}{l}\text { 'Often' } \\
(n=410)\end{array}$ & $\begin{array}{l}\text { 'Not often' } \\
(n=579)\end{array}$ & p-value & OR $[95 \% \mathrm{CI}]$ \\
\hline \multicolumn{5}{|l|}{ Discuss with patients ('often') } \\
\hline Diet & $179(43.7 \%)$ & $195(33.7 \%)$ & $<0.01$ & $1.5[1.2,2.0]$ \\
\hline Smoking and drug use & $96(23.5 \%)$ & $82(14.2 \%)$ & $<0.01$ & $1.9[1.3,2.6]$ \\
\hline Physical activity & $380(92.7 \%)$ & $504(87.0 \%)$ & $<0.01$ & $1.9[1.3,2.9]$ \\
\hline Occupation Health \& Safety & $232(56.7 \%)$ & $273(47.2 \%)$ & $<0.01$ & $1.4[1.1,1.9]$ \\
\hline Pain counselling & $90(22.0 \%)$ & $175(30.2 \%)$ & $<0.01$ & $0.7[0.5,0.9]$ \\
\hline Stress & $231(56.5 \%)$ & $257(44.5 \%)$ & $<0.01$ & $1.6[1.2,2.1]$ \\
\hline Nutrition & $122(29.8 \%)$ & $120(22.5 \%)$ & $<0.01$ & $1.5[1.1,1.9]$ \\
\hline Medication & $195(47.7 \%)$ & $196(33.9 \%)$ & $<0.01$ & $1.8[1.4,2.1]$ \\
\hline \multicolumn{5}{|l|}{ Patient presentations ('often') } \\
\hline Neck pain & $409(99.8 \%)$ & $560(96.7 \%)$ & $<0.01$ & $13.9[1.8,104.8]$ \\
\hline Thoracic pain & $397(96.8 \%)$ & $511(88.3 \%)$ & $<0.01$ & $4.1[2.2,7.5]$ \\
\hline Low back pain & $410(100 \%)$ & $565(97.8 \%)$ & $<0.01$ & $1.7[1.6,1.8]$ \\
\hline Hip musculoskeletal pain & $368(89.8 \%)$ & $374(64.7 \%)$ & $<0.01$ & $4.8[3.3,6.9]$ \\
\hline Knee musculoskeletal pain & $288(70.4 \%)$ & $202(34.9 \%)$ & $<0.01$ & $4.4[3.4,5.2]$ \\
\hline Ankle musculoskeletal pain & $217(53.0 \%)$ & $115(19.9 \%)$ & $<0.01$ & $4.5[3.4,6.0]$ \\
\hline Foot musculoskeletal pain & $191(4.6 . \%)$ & $102(17.6 \%)$ & $<0.01$ & $4.1[3.0,5.4]$ \\
\hline Shoulder musculoskeletal pain & $389(94.9 \%)$ & $411(71.1 \%)$ & $<0.01$ & $7.5[4.7,12.1]$ \\
\hline Elbow musculoskeletal pain & $182(44.6 \%)$ & $68(11.8 \%)$ & $<0.01$ & $6.1[4.4,8.3]$ \\
\hline Wrist musculoskeletal pain & $136(33.3 \%)$ & $51(8.8 \%)$ & $<0.01$ & $5.2[3.6,7.3]$ \\
\hline
\end{tabular}




\begin{tabular}{|c|c|c|c|c|}
\hline & $\begin{array}{l}\text { 'Often' } \\
(\mathrm{n}=410) \\
\end{array}$ & $\begin{array}{l}\text { 'Not often' } \\
(\mathrm{n}=579)\end{array}$ & p-value & OR $[95 \% \mathrm{CI}]$ \\
\hline Hand musculoskeletal pain & $94(23.0 \%)$ & $27(4.7 \%)$ & $<0.01$ & $6.1[3.8,9.5]$ \\
\hline Postural disorders & $337(82.4 \%)$ & $337(58.2 \%)$ & $<0.01$ & $3.3[2.5,4.5]$ \\
\hline Degenerative spine conditions & $315(77.0 \%)$ & $283(48.9 \%)$ & $<0.01$ & $3.5[2.6,4.7]$ \\
\hline Headache disorders & $387(94.4 \%)$ & $504(87.0 \%)$ & $<0.01$ & $2.5[1.5,4.1]$ \\
\hline Migraine disorders & $231(56.6 \%)$ & $169(29.1 \%)$ & $<0.01$ & $3.1[2.4,4.1]$ \\
\hline Spine health maintenance & $233(57.0 \%)$ & $225(38.9 \%)$ & $<0.01$ & $2.1[1.6,2.7]$ \\
\hline Chronic or persistent pain & $341(83.2 \%)$ & $288(49.8 \%)$ & $<0.01$ & $5.0[3.6,6.8]$ \\
\hline Temporomandibular joint disorders & $121(29.6 \%)$ & $62(10.7 \%)$ & $<0.01$ & $3.5[2.5,4.9]$ \\
\hline Non-musculoskeletal disorders & $61(15.1 \%)$ & $64(11.1 \%)$ & 0.07 & - \\
\hline \multicolumn{5}{|l|}{ Patient subgroups (treat 'often') } \\
\hline Up to 3 years of age & $62(15.2 \%)$ & $94(16.3 \%)$ & 0.64 & - \\
\hline 4 to 18 years of age & $142(34.6 \%)$ & $128(22.1 \%)$ & $<0.01$ & $1.9[1.4,2.5]$ \\
\hline Over 65 years of age & $298(72.7 \%)$ & $272(47.1 \%)$ & $<0.01$ & $3.0[2.3,3.9]$ \\
\hline $\begin{array}{l}\text { Aboriginal \& Torres Strait } \\
\text { Islander peoples }\end{array}$ & $3(0.7 \%)$ & $4(0.7 \%)$ & 0.94 & - \\
\hline Pregnancy & $187(45.6 \%)$ & $157(27.2 \%)$ & $<0.01$ & $2.2[1.7,2.9]$ \\
\hline Non-English speaking & $17(4.1 \%)$ & $16(2.8 \%)$ & 0.24 & - \\
\hline Sport injuries & $295(72.0 \%)$ & $203(35.2 \%)$ & $<0.01$ & $4.7[3.6,6.2]$ \\
\hline Worker injury (compensable) & $61(14.9 \%)$ & $41(7.1 \%)$ & $<0.01$ & $2.3[1.5,3.5]$ \\
\hline Work injury (non-compensable) & $197(48.0 \%)$ & $142(24.6 \%)$ & $<0.01$ & $2.8[2.1,3.7]$ \\
\hline Traffic injury (compensable) & $35(8.6 \%)$ & $18(3.1 \%)$ & $<0.01$ & $2.9[1.6,5.2]$ \\
\hline Traffic injury (non-compensable) & $81(19.9 \%)$ & $32(5.6 \%)$ & $<0.01$ & $4.2[2.7,6.5]$ \\
\hline Post-surgery & $54(13.2 \%)$ & $24(4.2 \%)$ & $<0.01$ & $3.5[2.2,5.7]$ \\
\hline \multicolumn{5}{|l|}{ Manual therapy (use 'often') } \\
\hline Counterstrain & $201(49.1 \%)$ & $217(37.5 \%)$ & $<0.01$ & $1.6[1.2,2.1]$ \\
\hline Muscle energy technique & $338(82.6 \%)$ & $447(77.2 \%)$ & 0.04 & $1.4[1.0,1.9]$ \\
\hline $\begin{array}{l}\text { High-velocity, low-amplitude } \\
\text { manipulation }\end{array}$ & $288(70.4 \%)$ & $342(59.1 \%)$ & $<0.01$ & $1.7[1.3,2.2]$ \\
\hline Joint manipulation & $209(51.2 \%)$ & $184(31.8 \%)$ & $<0.01$ & $2.2[1.7,2.9]$ \\
\hline Soft tissue technique & $368(90.0 \%)$ & $477(82.5 \%)$ & $<0.01$ & $1.9[1.3,2.8]$ \\
\hline Myofascial release & $282(68.9 \%)$ & $327(56.6 \%)$ & $<0.01$ & $1.7[1.3,2.2]$ \\
\hline Visceral techniques & $43(10.5 \%)$ & $55(9.5 \%)$ & 0.60 & - \\
\hline Lymphatic pump & $43(10.5 \%)$ & $41(7.1 \%)$ & 0.06 & - \\
\hline Autonomic balancing & $73(17.9 \%)$ & $84(14.5 \%)$ & 0.15 & - \\
\hline Biodynamics & $60(14.7 \%)$ & $95(16.4 \%)$ & 0.46 & - \\
\hline Functional technique & $125(30.6 \%)$ & $144(24.9 \%)$ & 0.04 & $1.3[1.0,1.7]$ \\
\hline Balanced ligamentous tension & $139(34.0 \%)$ & $209(36.1 \%)$ & 0.50 & - \\
\hline Chapman's reflexes & $17(4.2 \%)$ & $7(1.2 \%)$ & $<0.01$ & $3.6[1.4,8.6]$ \\
\hline Trigger point therapy & $126(30.8 \%)$ & $132(22.8 \%)$ & $<0.01$ & $1.5[1.1,2.0]$ \\
\hline Osteopathy in the Cranial Field & $88(21.6 \%)$ & $145(25.0 \%)$ & 0.20 & - \\
\hline Facilitated positional release & $83(20.3 \%)$ & $83(14.4 \%)$ & 0.01 & $1.5[1.1,2.2]$ \\
\hline Dry needling & $121(29.6 \%)$ & $113(19.6 \%)$ & $<0.01$ & $1.7[1.3,2.3]$ \\
\hline
\end{tabular}




\begin{tabular}{|c|c|c|c|c|}
\hline & $\begin{array}{l}\text { 'Often' } \\
(n=410)\end{array}$ & $\begin{array}{l}\text { 'Not often' } \\
(\mathrm{n}=579)\end{array}$ & p-value & OR $[95 \% \mathrm{CI}]$ \\
\hline Exercise prescription & $338(82.8 \%)$ & $393(67.9 \%)$ & $<0.01$ & $2.3[1.7,3.2]$ \\
\hline Shockwave therapy & $14(3.4 \%)$ & $4(0.7 \%)$ & $<0.01$ & $5.1[1.6,15.6]$ \\
\hline Ultrasound & $18(4.4 \%)$ & $9(1.6 \%)$ & $<0.01$ & $2.9[1.3,6.5]$ \\
\hline TENS & $14(3.4 \%)$ & $5(0.9 \%)$ & $<0.01$ & $4.1[1.4,11.4]$ \\
\hline Instrument manipulation & $1(0.2 \%)$ & $1(0.2 \%)$ & 0.81 & - \\
\hline Instrument soft-tissue & $9(2.2 \%)$ & $3(0.5 \%)$ & 0.02 & $4.3[1.1,16.1]$ \\
\hline Sport taping & $74(18.1 \%)$ & $48(8.3 \%)$ & $<0.01$ & $2.5[1.6,3.6]$ \\
\hline \multicolumn{5}{|l|}{ Expanded practice scope ('definitely') } \\
\hline Prescribing rights & $123(30.1 \%)$ & $133(23.0 \%)$ & 0.01 & $1.4[1.1,1.9]$ \\
\hline $\begin{array}{l}\text { Referral rights } \\
\text { to orthopaedic surgeon }\end{array}$ & $306(74.8 \%)$ & $394(68.0 \%)$ & 0.02 & $1.4[1.0,1.8]$ \\
\hline Referral rights to paediatrician & $231(56.5 \%)$ & $308(53.2 \%)$ & 0.31 & - \\
\hline $\begin{array}{l}\text { Referral rights to sports } \\
\text { medicine specialist }\end{array}$ & $333(81.6 \%)$ & $454(78.4 \%)$ & 0.21 & - \\
\hline Referral rights to rheumatologist & $287(70.2 \%)$ & $339(58.5 \%)$ & $<0.01$ & $1.6[1.3,2.2]$ \\
\hline $\begin{array}{l}\text { Referral rights to other } \\
\text { medical specialist }\end{array}$ & $1(0.3 \%)$ & 0 & 0.23 & - \\
\hline Expanded diagnostic imaging rights & $359(87.8 \%)$ & $461(79.6 \%)$ & $<0.01$ & $1.8[1.3,2.6]$ \\
\hline \multicolumn{5}{|l|}{ Research in practice ('strongly agree') } \\
\hline $\begin{array}{l}\text { Useful to help patients understand } \\
\text { the benefits of osteopathy }\end{array}$ & $182(44.4 \%)$ & $261(45.1 \%)$ & 0.83 & - \\
\hline $\begin{array}{l}\text { Useful to help GPs and other } \\
\text { conventional health professionals } \\
\text { understand the role of osteopathy }\end{array}$ & $280(70.9 \%)$ & $389(70.2 \%)$ & 0.82 & - \\
\hline $\begin{array}{l}\text { Useful to provide scientific } \\
\text { evidence for what I do } \\
\text { as an osteopath }\end{array}$ & $213(54.9 \%)$ & $301(54.9 \%)$ & 0.99 & - \\
\hline $\begin{array}{l}\text { Irrelevant to the professional } \\
\text { development of osteopathy } \\
\text { in Australia. }\end{array}$ & $232(59.9 \%)$ & $331(60.5 \%)$ & 0.86 & - \\
\hline $\begin{array}{l}\text { Impact of research on practice } \\
\text { ('high impact') }\end{array}$ & $410(100 \%)$ & $579(100 \%)$ & 0.18 & - \\
\hline
\end{tabular}

Adjusted odd ratios for significant characteristics associated with often treating tendinopathies are described in table IV. Australian osteopaths who often treat patients with tendinopathies were more likely to treat patients with chronic patients and sports injuries, but less likely to be involved in research.

\section{DISCUSSION}

There is limited literature on how osteopaths manage tendinopathies (18), and none describing the practice and clinical management characteristics associated with patients presenting with this complaint. Our work offers the first substantive description of how Australian osteopaths manage tendinopathies based on data from a large, nationally representative sample of practitioners (16).

The strongest association identified in the regression model for Australian osteopaths who often treat patients presenting with tendinopathies was with frequency of management of those patients with chronic or persistent pain (ORa 3.8). Whether this refers to chronic tendinopathies or more broadly, chronic musculoskeletal pain, is unclear. However, we posit that these osteopaths may be more likely to manage chronic tendinopathies when compared to their osteopathy counterparts who do not often treat this complaint. Previous work has shown chronic musculoskeletal pain is linked with 
Table IV. Adjusted odds ratios for significant characteristics of Australian osteopaths who often treat patients with tendinopathies.

\begin{tabular}{|c|c|c|c|}
\hline & $\begin{array}{c}\text { Adjusted } \\
\text { Odds Ratio }\end{array}$ & $\begin{array}{c}95 \% \text { Confidence } \\
\text { Interval }\end{array}$ & p-value \\
\hline \multicolumn{4}{|l|}{ Practitioner characteristics } \\
\hline Gender (female) & 1.5 & $1.0-2.1$ & 0.01 \\
\hline Involved in research (yes) & 0.5 & $0.2-0.9$ & 0.04 \\
\hline \multicolumn{4}{|l|}{ Conditions treated } \\
\hline Treat foot musculoskeletal complaints (often) & 1.7 & $1.1-2.7$ & $<0.01$ \\
\hline Treat shoulder musculoskeletal complaints (often) & 2.8 & $1.6-4.9$ & $<0.01$ \\
\hline Treat elbow musculoskeletal complaints (often) & 2.1 & $1.4-3.3$ & $<0.01$ \\
\hline Treat degenerative spine conditions (often) & 1.8 & $1.2-2.5$ & $<0.01$ \\
\hline Treat temporomandibular joint disorders (often) & 1.7 & $1.1-2.7$ & 0.02 \\
\hline \multicolumn{4}{|l|}{ Patient subgroups } \\
\hline Treat patients with chronic pain (often) & 3.8 & $2.6-5.5$ & $<0.01$ \\
\hline Treat patients with sports injuries (often) & 2.5 & $1.8-2.4$ & $<0.01$ \\
\hline Treat patients aged 65 years and older (often) & 1.7 & $1.2-2.4$ & $<0.01$ \\
\hline Treat compensable workers compensation patients (often) & 1.7 & $1.2-3.5$ & $<0.01$ \\
\hline \multicolumn{4}{|l|}{ Patient management } \\
\hline Use soft tissue techniques (often) & 2.1 & $1.2-3.5$ & 0.02 \\
\hline Use exercise prescription (often) & 1.6 & $1.0-2.4$ & 0.03 \\
\hline
\end{tabular}

cognitive, psychological and social factors that are recommended to be managed via a biopsychosocial (BPS) model of care (19). Australian osteopaths who often treat tendinopathies were approximately 50\% more likely to refer to psychologists, specialists and exercise physiologists, suggesting that osteopaths may manage tendinopathy patients using a BPS model. Further, Australian osteopaths who often treat tendinopathies are more likely to report receiving referrals from other healthcare professionals such as medical specialists, podiatrists and dieticians. This data suggests that Australian osteopaths may participate in multidisciplinary care of tendinopathies however such an assertion requires additional investigation.

Evidence-based approaches to tendinopathy recommend the use of exercise in its management, regardless of anatomical location (20-23). Australian osteopaths who often treat tendinopathies were $60 \%$ more likely to use exercise prescription in patient care compared to those osteopaths who reported not often treating this condition. Whether prescribed exercises accord with current evidence requires further exploration however the result is encouraging from the standpoint of osteopaths utilising this in patient care. Taping was also more likely to be used by those Australian osteopaths who often manage tendinopathies. Again, the specifics of this taping cannot be gleaned from our work, nor the type of taping used (e.g. rigid sports taping, kinesiology taping). However, given the equivocal nature of the literature with respect to taping for tendinopathies (23), the use and clinical reasoning for this intervention would be useful to explore.

Australian osteopaths who often treat tendinopathies are more likely to report often managing conditions effecting the extremities compared to those who do not often treat this complaint. In particular, those osteopaths who treat foot, shoulder and elbow musculoskeletal complaints were $50 \%$ more likely to report often treating tendinopathies compared to colleagues who do not often treat this complaint. Tendinopathies affecting the Achilles (2-4) and shoulder (5) are common presentations in the broader population and our results may be a reflection of these being a common presentation to Australian osteopaths. Further, our results also suggest that older patients are more likely to be treated often by osteopaths who often treat tendinopathies. The prevalence of tendinopathies in older patients, particularly those in the hip and shoulder, and sports injury populations has previously been reported (24). These outcomes highlight the role that Australian osteopaths may play in the management of tendinopathies in sport injury and older adult populations, providing an interesting area for additional research. 
The predominant treatment approach utilised in Australian osteopathy practice is manual therapy (16). Unadjusted odds ratios suggest Australian osteopaths who often treat tendinopathies are more likely to use a range of manual therapy interventions, including some not traditionally described in tendinopathy management (i.e., counterstrain, facilitated positional release). On this basis, we propose that osteopaths may utilise a range of management approaches for tendinopathies, potentially to modulate pain levels (25). Modulating pain may offer an avenue to explore exercise rehabilitation and other active management strategies. This strategy has been described in a case report describing the osteopathy management of Achilles tendinopathy with successful outcomes (18). How the use of these other manual therapy techniques or combinations of techniques contributes to patient outcomes, and the clinical reasoning of osteopaths with respect to technique choice in tendinopathy care would require further research.

\section{Limitations}

The cross-sectional and self-report nature of the design of the ORION questionnaire is a limitation when interpreting the results of the study. Cross-sectional self-report designs are potentially susceptible to social desirability bias and recall bias. How practitioners defined sometimes and often for example, when completing the questionnaire is open to interpretation and may skew the results. Data collection took place in mid-late 2016 and it is possible that the practice profile and characteristics have since changed. This may limit the generalisability of the work to contemporary osteopathy practice as the education providers have changed their curricula to include development of skills and knowledge with respective to active approaches (i.e., load management in exercise prescription) and pain education interventions. The design of the survey also does not allow us to identify the prevalence of specific tendinopathies nor the treatment approaches for these. It may be that practitioners approach different regions in different ways and this clinical reasoning would be valuable to explore.

\section{Future work}

As described, there are opportunities to explore the multidisciplinary nature of tendinopathy management where an osteopath is involved in the care team. How individual osteopaths clinically reason their management of a patient with a tendinopathy also would be of value. The role of adjunctive therapies such as dry needling and shockwave therapy as part of the approach osteopaths use for tend- inopathy also requires additional research. Osteopaths who often treat tendinopathies also appear to be engaging in both active (i.e., exercise prescription) and passive (i.e., manual therapy, SWT) interventions more frequently than colleagues who do not often treat this complaint. Again, whether these use of active and passive interventions is related to tendinopathy care could be the basis for future research. Additionally, future research should evaluate patient outcomes and cost-effectiveness of osteopathy care when managing tendinopathies. Broadly, the outcomes of the study suggest that the clinical management of tendinopathies by Australian osteopaths is focused on manual therapy combined with exercise, within a BPS approach to patient care. As highlighted, how this approach translates to care outcomes requires further evaluation in case-control and cohort designs.

\section{CONCLUSIONS}

Little is known about how tendinopathies are managed by osteopaths however this study begins to illuminate the approaches to patient management and practices of osteopaths who report often treating this musculoskeletal complaint. Tendinopathies are reported to be commonly managed by osteopaths in our study, with foot, elbow and shoulder musculoskeletal complaints, in addition to working with older and sport injury populations, being associated with those practitioners who often treat tendinopathies. These findings appear to be relatively consistent with our understanding of the epidemiology of tendinopathies with respect to gender, influence of sporting activity on the development of tendinopathies, and the regions of the body most commonly affected by this musculoskeletal issue. This study contributes data to the wider discourse about the management of tendinopathies in manual therapy practice and the role osteopaths may be able to play in the care of acute and chronic tendinopathies.

\section{ACKNOWLEDGEMENTS}

The ORION project is funded by Osteopathy Australia. The funding source had no influence in the design of the study and collection, analysis, and interpretation of data and in writing the manuscript. The research reported in this paper is the sole responsibility of the authors and reflects the independent ideas and scholarship of the authors alone. The authors would also like to thank Professor Jon Adams, Dr Amie Steel and Dr Wenbo Peng from the Australian Research Centre in Complementary and Integrative Medicine, University of Technology Sydney for their input into the initial stages of the analysis and data interpretation. 


\section{CONFLICT OF INTERESTS}

The authors declare that they have no conflict of interests.

\section{REFERENCES}

1. Xu Y, Murrell GA. The basic science of tendinopathy. Clin Orthop Relat Res 2008;466:1528-1538.

2. Cardoso TB, Pizzari T, Kinsella R, Hope D, Cook JL. Current trends in tendinopathy management. Best Pract Res Clin Rheumatol 2019;33:122-140.

3. de Jonge S, Van den Berg C, de Vos R-J, et al. Incidence of midportion Achilles tendinopathy in the general population. Br J Sports Med 2011;45:1026-1028.

4. Cassel M, Risch L, Intziegianni K, et al. Incidence of Achilles and patellar tendinopathy in adolescent elite athletes. Int J Sports Med 2018;39:726-732.

5. Littlewood C, May S, Walters S. Epidemiology of rotator cuff tendinopathy: a systematic review. Shoulder \& Elbow 2013;5:256-265.

6. Stephens G, O'Neill S, French HP, et al. A survey of physiotherapy practice (2018) in the United Kingdom for patients with greater trochanteric pain syndrome. Musculoskeletal Sci Pract 2019;40:10-20.

7. Albers IS, Zwerver J, Diercks RL, Dekker JH, Van den AkkerScheek I. Incidence and prevalence of lower extremity tendinopathy in a Dutch general practice population: a cross sectional study. BMC Musculo Disorder 2016;17:16.

8. Cassel M, Baur H, Hirschmüller A, Carlsohn A, Fröhlich K, Mayer F. Prevalence of Achilles and patellar tendinopathy and their association to intratendinous changes in adolescent athletes. Scand J Med Sci Sports 2015;25:e310-e318.

9. Zwerver J, Bredeweg SW, van den Akker-Scheek I. Prevalence of Jumper's knee among nonelite athletes from different sports: a cross-sectional survey. Am J Sports Med 2011;39:1984-1988.

10. Hopkins C, Fu S-C, Chua E, et al. Critical review on the socio-economic impact of tendinopathy. Asia Pac J Sports Med Arthro Rehabil Technol 2016;4:9-20.

11. Malliaras P, Barton CJ, Reeves ND, Langberg H. Achilles and Patellar Tendinopathy Loading Programmes. Sports Med 2013;43:267-286.

12. Martin RL, Chimenti R, Cuddeford T, et al. Achilles pain, stiffness, and muscle power deficits: Midportion achilles tendinopathy revision 2018: Clinical practice guidelines linked to the international classification of functioning, disability and health from the Orthopaedic Section of the American
Physical Therapy Association. J Orthop Sports Phys Ther 2018;48:A1-A38.

13. Mallows A, Debenham J, Walker T, Littlewood C. Association of psychological variables and outcome in tendinopathy: a systematic review. Br J Sports Med 2017;51:743-748.

14. Drake C, Mallows A, Littlewood C. Psychosocial variables and presence, severity and prognosis of plantar heel pain: A systematic review of cross-sectional and prognostic associations. Musculoskeletal Care 2018;16:329-338.

15. Plinsinga ML, Coombes BK, Mellor R, et al. Psychological factors not strength deficits are associated with severity of gluteal tendinopathy: A cross-sectional study. Eur J Pain 2018;22:1124-1133.

16. Adams J, Sibbritt D, Steel A, Peng W. A workforce survey of Australian osteopathy: analysis of a nationally-representative sample of osteopaths from the Osteopathy Research and Innovation Network (ORION) project. BMC Health Serv Res 2018;18:352.

17. Padulo J, Oliva F, Frizziero A, Maffulli N. Basic principles and recommendations in clinical and field science research: 2018 update. MLTJ 2018;8:305-307.

18. Ross G, Macfarlane C, Vaughan B. Combined osteopathy and exercise management of Achilles tendinopathy in an athlete. J Sports Med Phys Fitness 2018;58:106-112.

19. Sluka KA: Mechanisms and management of pain for the physical therapist: Lippincott Williams \& Wilkins, 2016.

20. Littlewood C, Malliaras P, Chance-Larsen K. Therapeutic exercise for rotator cuff tendinopathy: a systematic review of contextual factors and prescription parameters. Int J Rehabil Res 2015;38:95-106.

21. Grimaldi A, Mellor R, Hodges P, Bennell K, Wajswelner H, Vicenzino B. Gluteal tendinopathy: a review of mechanisms, assessment and management. Sports Med 2015;45:1107-1119.

22. Malliaras P, Cook J, Purdam C, Rio E. Patellar tendinopathy: clinical diagnosis, load management, and advice for challenging case presentations. J Orthop Sports Phys Ther 2015;45:887-898.

23. Wilson F, Walshe M, O'dwyer T, Bennett K, Mockler D, Bleakley C. Exercise, orthoses and splinting for treating Achilles tendinopathy: a systematic review with meta-analysis. Br J Sports Med 2018;52:1564-1574.

24. Kaux J-F, Forthomme B, Le Goff C, Crielaard J-M, Croisier J-L. Current opinions on tendinopathy. J Sports Sci Med 2011;10:238.

25. Voogt L, de Vries J, Meeus M, Struyf F, Meuffels D, Nijs J. Analgesic effects of manual therapy in patients with musculoskeletal pain: a systematic review. Man Ther 2015;20:250-256. 